D. Mendoza et al.

\title{
CONSUMO ELÉCTRICO, CONFORT TÉRMICO E IMPACTO AMBIENTAL DE UNA INSTITUCIÓN EDUCATIVA UBICADA EN EL NORTE DE ARGENTINA
}

\author{
ELECTRICITY CONSUMPTION, THERMAL COMFORT AND \\ ENVIRONMENTAL IMPACT OF AN EDUCATIONAL INSTITUTION \\ LOCATED IN THE NORTH OF THE ARGENTINA
}

Dora Mendoza-Vela ${ }^{11}{ }^{1}$, Cecilia Castro-Mendoza ${ }^{1}[$

Pablo Mendoza-Vela

'Sede Regional Orán de la Universidad Nacional de Salta, Ciudad de Orán, Prov. de Salta, Rep. Argentina

Recibido (Received): 22 / 01 / 2021 Aceptado (Accepted): 18/ 06 / 2021

\begin{abstract}
RESUMEN
El objetivo de este trabajo es conocer el consumo eléctrico de una institución educativa por el uso de equipos de aire acondicionado para el confort térmico, como también el impacto local y ambiental que genera dicho consumo. La institución se encuentra en la ciudad de Orán (Norte de Argentina), en zona bioclimática IIb (Norma IRAM 11603) y se trata de una Sede Universitaria. Para su funcionamiento dispone de 2 predios: el predio 1 se encuentra en zona céntrica y es la unidad de estudio. Aplicando diferentes técnicas e instrumentos se investiga acerca de las condiciones climáticas de la región, los aspectos estructurales y funcionales del edificio de la institución, la dinámica de la comunidad educativa y sus preferencias tecnológicas para el logro del confort térmico, el consumo eléctrico en el periodo 2013-2018 y las áreas de demanda eléctrica. Con todos los datos obtenidos y, aplicando un procedimiento basado en meses referentes, se estima el consumo eléctrico por el uso de equipos de aire acondicionado. También se analizan los impactos locales y se estima la huella de carbono por esa actividad. Los resultados más importantes son: se trata de una región muy calurosa, el aspecto estructural del edificio carece de conceptos bioclimáticos, el consumo eléctrico por el uso de equipos de aire acondicionado representa el $28 \%$ del consumo anual total del predio $1 \mathrm{y}$ una emisión aproximada de 13 tnCO 2 eq/año, requiriendo entre 1300 y 2600 árboles para su mitigación. Resulta imprescindible implementar estrategias bioclimáticas en el edificio de la Sede Universitaria.
\end{abstract}

Palabras Clave: Consumo eléctrico, Confort Térmico, Institución Educativa, Huella de Carbono.

\section{ABSTRACT}

The aim of this paper is to know the electricity consumption of an educational institution by the use of air conditioning equipment for thermal comfort, as well as the local and environmental impact that such consumption generates. The institution is located in the city of Orán (North of Argentina), in bioclimatic zone IIb (IRAM Standard 11603) and it is a University headquarters. For its operation it has 2 properties: property 1 is located in a downtown area and is the study unit. Applying different techniques and instruments is investigated about the climatic conditions of the region, the structural and functional aspects of the institution building, the dynamics of the educational community and its technological preferences for the achievement of thermal comfort, the electricity consumption in the period 2013-2018 and the areas of electricity demand. With all the data obtained and, applying a procedure based on reference months, the electricity consumption for the use of air conditioning equipment is estimated. Local impacts are also analyzed and the carbon footprint for this activity is estimated. The most important results are: it is a very hot region, the structural aspect of the building lacks bioclimatic concepts, the electricity consumption from the use of air conditioning equipment represents $28 \%$ of the total annual consumption of the property 1 and an approximate emission of 13 tnCO $\mathrm{O}_{2}$ eq / year, requiring between 1,300 and 2,600 trees for mitigation. It is essential to implement bioclimatic strategies in the building of the University Headquarters.

Keywords: Electricity Consumption, Thermal Comfort, Educational Institution, Carbon Footprint.

\section{INTRODUCCIÓN}

La ciudad de Orán se encuentra en una zona con clima cálido-húmedo (a $33 \mathrm{Km}$ del Trópico de (apricornio), con periodos estivales que abarcan desde mediados de setiembre a mediados de abril. Se caracteriza por sus registros de temperaturas absolutas mayores a $40{ }^{\circ} \mathrm{C}$, generando mucho malestar en toda la

\footnotetext{
${ }^{1}$ Corresponding author.:

E-mail: dmendoza412@gmail.com
} 


\section{Mendoza et al.}

comunidad. Por lo general, en el diseño de los edificios no se consideran las condiciones climáticas de la región ni otros principios bioclimáticos. Para el logro del confort térmico se recurren a los equipos de aire acondicionados (AAC): tanto las familias de bajos $y$ medianos ingresos se proveen de al menos un equipo. Así también lo hacen las instituciones educativas: la gran mayoría de ellas recurren a estos equipos para el desarrollo de sus actividades académicas y administrativas en ambientes confortables y su uso es intensivo durante dos o tres turnos diarios.

Desde ese abordaje, el objetivo de este trabajo es conocer el consumo eléctrico en que incurre una institución educativa por el uso de equipos de AAC para el logro del confort térmico. Así también se busca conocer el impacto local y ambiental que genera dicho consumo.

La institución educativa es una Sede de la Universidad Nacional de Salta que funciona de 7 a 22 hs. Dispone de 2 predios: el primero se encuentra en zona céntrica y el segundo en zona periférica. Antiguamente, el Predio 1 funcionaba como hospital, pero en 1981 fue cedido a la universidad. Por ello se tuvieron que realizar importantes remodelaciones, pero sólo considerando criterios funcionales.

Luego de aplicar diferentes técnicas e instrumentos de recogida de datos y procesar la información referente al predio 1 se determina: el consumo eléctrico medio anual, los consumos medios mensuales, las áreas de demanda de energía eléctrica, los consumos durante los meses estivales, las soluciones tecnológicas para el logro del confort térmico. Usando un procedimiento basado en meses referentes, se estima el consumo eléctrico por el uso de equipos de AAC. También se analizan la conformación de espacios contiguos confortables e inconfortables y la ausencia de estrategias bioclimáticas. Finalmente se estima la huella de carbono por esa actividad y se analiza una posible compensación para dichas emisiones.

La compensación de emisiones no debería ser la solución al problema del calentamiento global y cambio climático. Por el contrario, debería considerarse al diseño bioclimático como estrategia de prevención y reducción de emisiones de $\mathrm{CO}_{2}$.

\section{ANTECEDENTES}

\subsection{LOS EDIFICIOS Y SU RELACIÓN CON EL CONSUMO ENERGÉTICO}

Los edificios proporcionan un entorno de vida y de trabajo a los seres humanos: el $80 \%$ de la vida de las personas transcurre en el interior de los edificios. Durante el año 2004 solamente los edificios agotaron casi el 37\% de la energía del mundo, y se proyecta que alcance el 42 \% para el año 2030. En Europa, en el año 2000 , el $45 \%$ de la energía producida se usó en el sector edilicio y el $50 \%$ de la polución fue causada por el mismo sector [1].

El consumo edilicio está fuertemente relacionado con el comportamiento de los habitantes, cuyas demandas energéticas están sujetas a las temperaturas ambientales exteriores. En Argentina, el consumo eléctrico suele incrementarse en un $50 \%$ en época de verano, valor que podría reducirse hasta un $76 \%$ si se aplicaran políticas efectivas de fomento a la eficiencia energética y de planificación urbana [2].

Para el año 2002 la demanda mundial de energía primaria fue de 10435 GTOE (Giga Toneladas equivalentes en Petróleo) y las proyecciones anuncian que la demanda de energía primaria se duplicará en el año 2037 y se triplicará hacia el 2057 [3]. Resulta imprescindible pensar y planificar edificios sustentables, con un diseño eficiente, que usen la mínima energía para el confort de sus habitantes y de ser posible que provengan de fuentes renovables y limpias. Las cuestiones energéticas deben formar parte desde el inicio del proyecto.

\subsection{EL EFECTO INVERNADERO Y LA INCIDENCIA DE LOS EDIFICIOS}

El efecto invernadero es el fenómeno natural que permite a la tierra mantener las condiciones necesarias para albergar vida. La atmósfera captura algunos de los rayos del sol que llegan a la corteza terrestre, manteniéndolos dentro para conseguir una temperatura media de $15{ }^{\circ} \mathrm{C}$, caso contrario la misma sería de $-18{ }^{\circ} \mathrm{C}$. La atmósfera está compuesta por diversos gases naturales. En las proporciones adecuadas estos gases cumplen su función, pero al aumentar su concentración la atmósfera retiene más calor de lo necesario, provocando aumento de temperaturas, deshielos de los polos, desertificación, incendios, tormentas e inundaciones [4].

Hay tres razones principales por las que aumentan los Gases de Efecto Invernadero (GEI): la quema de combustibles fósiles (petróleo, carbón y gas natural), la deforestación de bosques y destrucción de ecosistemas (que absorben el $\mathrm{CO}_{2}$ ) y el aumento de la población, principalmente en zonas urbanas.

En los últimos mil años, la emisión de $\mathrm{CO}_{2}$ se mantuvo por debajo de las 300 ppm (partes por millón), mientras que a mediados del siglo XX comenzó su ascenso vertiginoso (a la par de la revolución industrial) y hoy en día ya ha superado ampliamente las 400 ppm [5]. Estas emisiones están directamente relacionadas con la temperatura media del aire (Fig. 1). 
D. Mendoza et al.

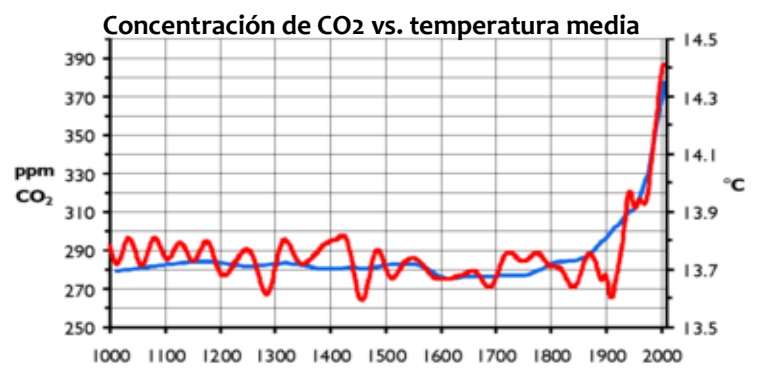

Fig.1. Concentración de $\mathrm{CO}_{2}$ en la atmósfera terrestre (línea azul) y temperatura media global en los últimos mil años (línea roja). [5]

La influencia humana en el sistema climático es clara: alrededor de la mitad de las emisiones antropogénicas de $\mathrm{CO}_{2}$ acumuladas entre 1750 y 2011 se han producido en los últimos 40 años (Fig. 2).

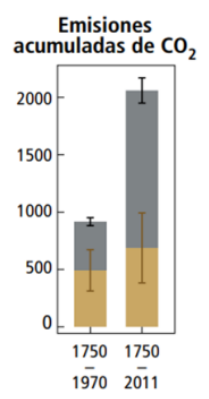

Fig.2. Emisiones acumuladas de $\mathrm{CO}_{2}$ desde 1750 a 2011. [5]

Se tratan de emisiones procedentes de la combustión de combustibles fósiles, la producción de cemento y quema en antorcha, la silvicultura y otros usos del suelo (Fig.3).

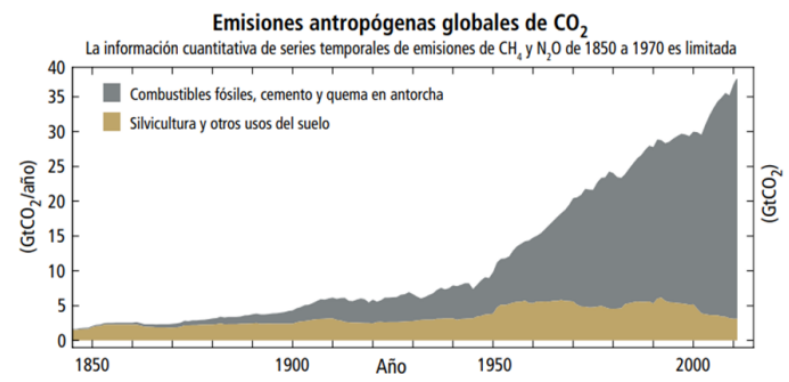

Fig.3. Emisiones antropogénicas globales anuales de $\mathrm{CO}_{2}$ procedentes de la combustión de combustibles fósiles, la producción de cemento y la quema en antorcha, y la silvicultura y otros usos del suelo, de 1750 a 2011. [5]

Los edificios tienen una participación importante en la emisión de $\mathrm{CO}_{2}$ : durante la producción de los materiales y su transporte, durante la construcción del edificio y a lo largo de su vida útil por el uso de gas, electricidad y carbón, entre otros, para cocina, agua sanitaria, iluminación y calefacción de ambientes. Al ritmo actual, se proyecta que en América Latina y otras regiones del mundo las emisiones de $\mathrm{CO}_{2}$ se duplicarán (Fig. 4) [6]-[7].

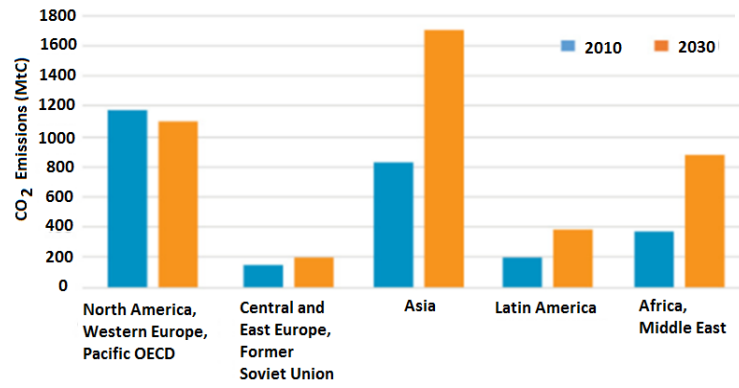

Fig. 4. Emisiones actuales y proyectadas de $\mathrm{CO}_{2}$ por región mundial. [7]

Argentina se ubica el puesto 22 del ranking mundial de las 192 naciones que son parte de la Convención Marco de las Naciones Unidas sobre el Cambio Climático [8], con una emisión de GEI de 0,9\% a escala planetaria. Dichas emisiones (Fig. 5) provienen un $51 \%$ del sector agropecuario y como consecuencia de la deforestación, un $23 \%$ por la producción energética, otros [9].

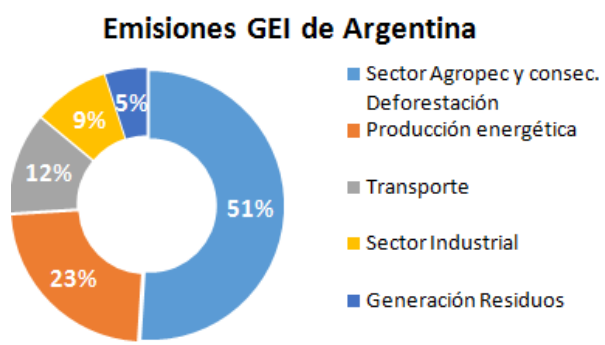

Fig. 5. Emisiones de GEI de la Argentina por sectores. [9]

En la Conferencia de Naciones Unidas para el Cambio Climático, París-COP21, se acordó acciones que eviten el incremento de la temperatura media de la superficie terrestre por encima de $2{ }^{\circ} \mathrm{C}$, como también incrementar estrategias que posibiliten la adaptación de la humanidad a estos cambios [10]-[11]. Desde la arquitectura bioclimática es posible reducir el consumo energético de los edificios y con ello el impacto ambiental generado [5].

\subsection{LAS EMISIONES DE CARBONO EN TIEMPOS DE COVID-19}

Según la investigación científica United in Science, llevada a cabo por la Organización Meteorológica Mundial (OMM), el Programa de la ONU para el Medio Ambiente, el Panel Intergubernamental de Expertos del Cambio Climático (IPCC), la UNESCO y otras organizaciones internacionales, se estima que el quinquenio 2016-2020 será el más cálido de la historia registrada, tendencia que se mantendrá en los años siguientes [12].

Debido a la pandemia por COVID-19, y a las medidas de confinamiento en todo el mundo por varios meses, las emisiones de GEI se redujeron sutilmente y 
D. Mendoza et al.

en la actualidad ya están regresando a los niveles anteriores. A pesar de este leve descenso, se está muy lejos de cumplir con los objetivos del Acuerdo de Paris. Las estaciones de referencia de la red de Vigilancia Global de la Atmósfera de la OMM informaron que para el mes de julio del año 2020 las estaciones de Mauna Loa (Hawai) y el cabo Grim (Tasmania) registraron concentraciones de 414,38 ppm y 410,04 ppm, respectivamente, frente a las 411,74 ppm y 407,83 ppm del mismo mes en 2019 [13].

\subsection{HUELLA DE CARBONO}

La Huella de Carbono (HC) es un instrumento que permite estimar las emisiones de GEI emitidos por un individuo, organización, evento o producto. El cálculo consiste en recopilar los datos referentes a los consumos directos e indirectos de insumos materiales (ej: papel) y energía, y traducirlos en emisiones de $\mathrm{CO}_{2}$ equivalentes [14].

La HC promedio en la Argentina es 5,71 tn/año por habitante como resultado del uso de energía, transporte, alimentación, hábitos de consumo y de esparcimiento, otros. Este valor es mayor al promedio mundial de 4 tn/año por habitante, pero menor al de Estados Unidos y del Reino Unido, 20 tn y 11,8 th respectivamente [15].

Según la norma PAS 2050:2011, el cálculo de la HC contempla los siguientes pasos:

1) Alcance de la medición, definiendo periodo del cálculo y límites del sistema a evaluar

2) Recolección de los datos e identificación de los Factores de emisión

3) Cálculo, considerando los flujos de entrada y salida de acuerdo a la unidad funcional y aplicando el producto entre el consumo eléctrico en un año y el Factor de emisión

4) Resultados y oportunidad de reducción. [14]:

Algunos Factores de emisión y sus unidades son

- $\quad$ Energía eléctrica: 0,486 $\mathrm{kgCO}_{2} \mathrm{eq} / \mathrm{kWh}$

Nafta: $2,37 \mathrm{kgCO}_{2}$ eq/litro

Gas natural: 1,95 $\mathrm{kgCO}$ eq $/ \mathrm{m}^{3}$

Papel Resma A4-75gr: $1320 \mathrm{kgCO}_{2} \mathrm{eq} / \mathrm{tn}$.

\subsection{REDUCCIÓN VS. COMPENSACIÓN}

Las estrategias de reducción tienen como objetivo disminuir la emisión de $\mathrm{CO}_{2}$ al ambiente y así disminuir el calentamiento global y sus consecuencias. Algunas a considerar son [16]:

- Eficiencia energética: apagado de artefactos que no se utilizan, reemplazo por tecnología de bajo consumo, mejora de aislamientos térmicos en las instalaciones, otros
- Eficiencia en el uso de recursos materiales (por ejemplo: uso consciente del papel, separación de residuos, reciclados)

- Transporte sustentable

- Uso de energías renovables

- $\quad$ Educación energética.

Si bien la prevención y reducción de las emisiones son el punto clave en el rol activo de la sociedad frente al Cambio Climático, es importante también mencionar que las emisiones pueden compensarse (aumentar el secuestro del carbono) mediante estrategias y medidas que aumenten la masa fotosintética, es decir captura de $\mathrm{CO}_{2}$ y liberación de $\mathrm{O}_{2}$ [16]. Algunas medidas son: plantación de árboles, promoción de huertas colectivas en espacios comunitarios urbanos, implementación de muros verticales, techos y terrazas verdes, entre otras.

Cada especie de árbol tiene una capacidad de absorción de $\mathrm{CO}_{2}$ diferente, su fijación varía en función de la especie y de las condiciones ambientales en las que se desarrollan. Se estima que un árbol absorbe entre 5 a $10 \mathrm{kgCO}_{2} /$ año [17].

\section{METODOLOGÍA}

\subsection{UNIDAD DE ESTUDIO}

La unidad de estudio es una Sede de la Universidad Nacional de Salta, que se encuentra en la ciudad de Orán, Provincia de Salta (Argentina). Como la misma dispone de dos predios, el estudio se focaliza en el Predio 1, ubicado en el casco céntrico de la ciudad.

\subsection{OBJETIVO DEL TRABAJO}

El objetivo del trabajo es conocer el consumo eléctrico en el que incurre una Institución educativa para el logro del confort térmico en época de verano (especialmente en periodos de altas temperaturas), como también identificar los impactos locales y ambientales que se generan a raíz de dicho consumo.

\subsection{ELEMENTOS METODOLÓGICOS}

Para lograr el objetivo se llevan a cabo las siguientes actividades:

1) Relevamiento de condiciones climáticas de la zona

2) Selección de una institución educativa, estudio de diferentes aspectos básicos (ubicación, orientación, estructural, funcional) y de su comunidad educativa

3) Identificación de áreas de demanda energética y procesamiento de datos sobre consumo eléctrico

4) Identificación de equipos tecnológicos más comunes usados por la comunidad educativa para el logro del confort térmico 
5) Estimación del consumo eléctrico destinado al logro del confort térmico

6) Análisis de los impactos locales y ambientales que se generan a causa de logro del confort térmico dentro del predio 1.

\section{ANÁLISIS DE RESULTADOS}

\subsection{LA CIUDAD DE ORÁN: CLIMA Y PROVISIÓN DE ENERGÍA ELÉCTRICA}

La ciudad de Orán se encuentra a $50 \mathrm{~km}$ de la frontera con la República de Bolivia, está inmersa en el Valle de Zenta y rodeada de cerros de la precordillera de los Andes. Sus coordenadas son $23^{\circ} 08^{\prime} 10^{\prime \prime}$ latitud S y $64^{\circ} 19^{\prime} 20^{\prime \prime}$ longitud O, a $336 \mathrm{msnm}$.

De acuerdo a la zonificación bioclimática de la Argentina (Norma IRAM 11603), la ciudad se encuentra en la zona IIb, con clima cálido-húmedo. Según información estadística provista por el Servicio Meteorológico Nacional (SMN) para el periodo 19812010 [18]-[19], el periodo estival se extiende desde octubre a marzo, con temperatura máxima media de $31,5{ }^{\circ} \mathrm{C}$ para esos meses. Los valores medios mensuales de humedad relativa rondan entre $59 \%$ y $83 \%$ y la velocidad media del viento entre 6,2 y 9,6 km/h . Las temperaturas máximas medias y máximas absolutas para los meses setiembre a diciembre, periodo 20162019, permiten apreciar las jornadas calurosas que caracterizan a esta zona (Fig. 6).

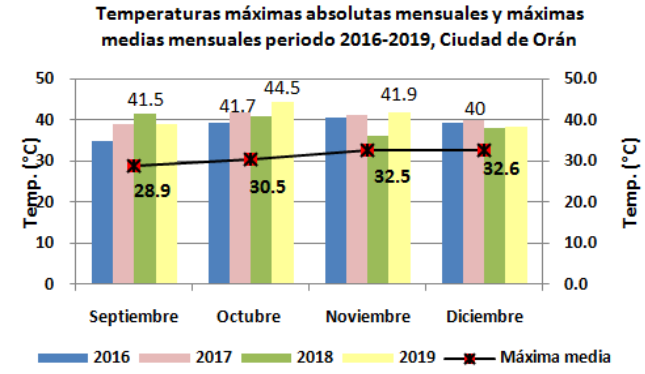

Fig. 6. Temperaturas máximas absolutas mensuales y máximas medias mensuales, periodo 2016-2019, Orán.

Por su latitud y condiciones climáticas, el recurso solar está disponible prácticamente todo el año. Para Orán, los valores estimados de radiación solar global acumulada anual sobre plano horizontal rondan entre 1700 y $1800 \mathrm{KWh} / \mathrm{m}^{2}$ año con valores mensuales promedios de entre $2,31 \mathrm{KWh} / \mathrm{m}^{2}$ para junio y 6,83 $\mathrm{KWh} / \mathrm{m}^{2}$ para diciembre [20].

El Sistema Eléctrico Nacional en Argentina tiene tres actores independientes: generadores, transportadores y distribuidores. Para el caso de Orán, los generadores pueden provenir de cualquier lado por estar interconectados. A ello también se pueden sumar las conexiones de la Central Térmica de Orán y las reservas frías de la propia ciudad. El transportador es TRASNOA y el distribuidor es EDESA.

El incremento demográfico de las últimas décadas y las configuraciones urbanas por el efecto de la Isla de Calor Urbana [21], han llevado a que la provisión de energía eléctrica en la ciudad resulte insuficiente. La demanda ha crecido notablemente y se acentúa en cada verano principalmente el uso intensivo de AAC.

\subsection{LA INSTITUCIÓN EDUCATIVA: ASPECTOS ESTRUCTURALES Y FUNCIONALES}

En la actualidad, la Sede Universitaria dispone de dos predios: el Predio 1 ubicado en zona céntrica, y el Predio 2 ubicado en zona periférica. El Predio 1 está rodeado con edificios públicos importantes, las calles son de pavimento y con mucha circulación vehicular.

Inicialmente la institución fue creada sin edificio propio y recién en el año 1981 obtuvo uno: las instalaciones de un viejo hospital construido entre 1917 y 1940 [22]. El acondicionamiento requirió remodelaciones importantes a fin de ajustarse a las necesidades académicas y administrativas:

- Remodelaciones (sector quirúrgico en un Salón Auditorium, consultorios en boxes docentes y oficinas)

Demoliciones (capilla y morgue, actualmente espacios destinados a accesos peatonal $y$ vehicular)

- Demolición del tercer piso de uno de los edificios y construcción de aulas en el sector del fondo.

Con respecto a lo funcional, el predio 1 cuenta con un sector administrativo (10 oficinas y salón de reuniones), 8 aulas amplias de uso general, aulas de uso específico (laboratorios, talleres, salas informáticas), unidades de investigación (de Enfermedades Tropicales, Robótica, Energías Renovables), biblioteca, 15 boxes docentes, Centro de Estudiantes, sector de servicio y taller para el personal de obras y servicios.

En relación a lo estructural, el predio 1 abarca un área de 3117,45 m² (orientación Norte-Sur), con un $82 \%$ de superficie edificada que concentra cinco edificios contiguos distribuidos horizontalmente (tres de NorteSur y dos Este-Oeste). Sólo uno de los edificios dispone de dos plantas. El $90 \%$ de las paredes externas son de $30 \mathrm{~cm}$ (mampostería de ladrillo macizo de 12,5 x 25,0 cm con revoque grueso y fino en exterior e interior), sin aislamiento térmico. Las paredes internas son de $20 \mathrm{~cm}$. Todas las paredes son de colores claros. En relación a los techos, el $7 \%$ tiene tejas, el $17 \%$ es losa + membrana asfáltica con aislación hidrófuga, y el resto es de chapas de zinc + cielorraso. La superficie parquizable es escasa. 
D. Mendoza et al.

En relación a las puertas y aberturas, todas las ventanas tienen vidrio común, además el $36 \%$ tiene celosías y el $35 \%$ cortinas blancas. Por lo general las mismas permanecen cerradas ya sea por confort térmico, acústica y limpieza. Es poco frecuente la ventilación nocturna por razones de seguridad. En relación a las puertas, el $60 \%$ son de madera maciza, mientras que las restantes son de placas. Por lo general permanecen cerradas durante el dictado de clases.

\subsection{LA COMUNIDAD EDUCATIVA Y LAS SOLUCIONES TECNOLÓGICAS PARA EL CONFORT TÉRMICO}

A la sede asisten alrededor de 1900 alumnos y 170 trabajadores (personal docente y no docente), pero sólo el $35 \%$ de los alumnos y el $\mathbf{6 0} \%$ del personal docente y no docente asisten al Predio 1.

La población estudiantil es muy heterogénea en edad, nivel socio-cultural y procedencia (localidades vecinas). Los alumnos permanecen en el recinto de la sede desde horas tempranas hasta el anochecer. Sus necesidades energéticas están relacionadas con la carga de baterías de Notebooks y celulares. Los consumos grupales están relacionados con lo requerido en las clases: iluminación, confort térmico, recursos didácticos, otros.

Las soluciones actuales para el logro del confort térmico en el Predio 1 se basan principalmente en el uso de equipos de AAC. Cada espacio de trabajo dispone de por lo menos un equipo de entre 3000 a 15000 frigorías. Sin embargo, sólo algunas oficinas disponen de estufas eléctricas y su uso es eventual.

\subsection{CONSUMO ELÉCTRICO DEL PREDIO 1 PARA EL PERIODO 2013-2018}

Como la sede funciona de Febrero a Diciembre con receso de dos semanas en Julio, el consumo eléctrico mínimo se da en Enero. Analizando el consumo mensual de cada año para el periodo 2013-2018 se tiene que (Fig. 7):

En los periodos de receso (Enero y Julio) los consumos mensuales son mínimos: 4,5 a $5 \mathrm{MWh}$

Los mayores consumos medios mensuales se dan en Octubre y Noviembre: alrededor de $11 \mathrm{MWh}$

El Consumo Medio Anual es $96 \mathrm{MWh}$.

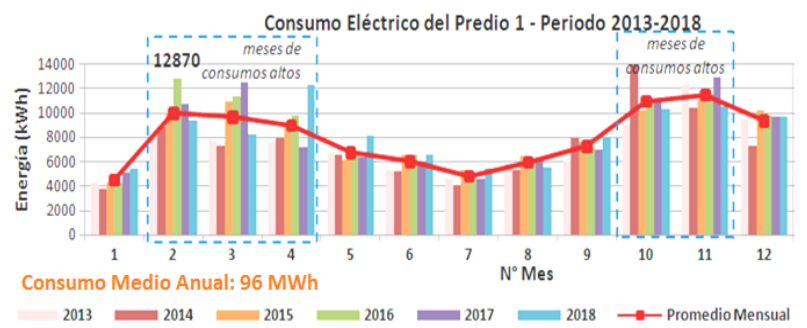

Fig. 7 Consumo eléctrico mensual de cada año y promedios mensuales del periodo 2013-2018 para el Predio 1.

Con esta información es posible predecir el comportamiento del consumo eléctrico mensual y anual del Predio 1 para los siguientes años, tanto en condiciones normales de funcionamiento como en años especiales. Por ejemplo, para el año 2020, con situación de pandemia, se estima que el consumo anual sea del $50 \%$ del Consumo Medio Anual.

Al tratarse de una institución dedicada a la tarea educativa, las áreas de demanda eléctrica difieren levemente de las de una vivienda (Fig. 8).

\begin{tabular}{|c|c|c|c|c|c|c|c|c|c|c|c|c|}
\hline \multirow{2}{*}{$\begin{array}{l}\text { Áreas de demanda } \\
\text { eléctrica en el } \\
\text { Predio } 1 \text { de la Sede } \\
\text { Univ. }\end{array}$} & \multicolumn{12}{|c|}{ Año Lectivo } \\
\hline & 1 & 2 & 3 & 4 & 5 & 6 & 7 & 8 & 9 & 10 & 11 & 12 \\
\hline \multicolumn{13}{|l|}{ 1- Iluminación } \\
\hline \multicolumn{13}{|l|}{$2-$ Red } \\
\hline \multicolumn{13}{|l|}{ 3-Informática } \\
\hline \multicolumn{13}{|l|}{ 4-Telefonía } \\
\hline \multicolumn{13}{|l|}{$\begin{array}{l}\text { 5- Multimedia para } \\
\text { la tarea docente }\end{array}$} \\
\hline \multicolumn{13}{|l|}{$\begin{array}{l}6 \text { - Provisión de } \\
\text { agua caliente }\end{array}$} \\
\hline \multicolumn{13}{|l|}{$\begin{array}{l}\text { 7-Confort Térmico: } \\
\text { Refrigeración }\end{array}$} \\
\hline \multicolumn{13}{|l|}{$\begin{array}{l}\text { 7-Confort Térmico: } \\
\text { Calefacción }\end{array}$} \\
\hline \multicolumn{13}{|l|}{ 8-Otros } \\
\hline $\begin{array}{l}\text { Consumo Medio } \\
\text { Mensual del } \\
\text { periodo 2013-2018 } \\
\text { (KWh) }\end{array}$ & 4535 & 9990 & 9695 & 9000 & 6740 & 6045 & 4813 & 5950 & 7295 & 11010 & 11480 & 9324 \\
\hline
\end{tabular}

Fig. 8. Esquema de las áreas de consumo eléctrico y los consumos medios mensuales durante el funcionamiento del Predio 1 , para el periodo 2013-2018.

\subsection{CONSUMO ELÉCTRICO POR EL USO DE EQUIPOS DE AAC}

Para identificar el consumo eléctrico por el uso de equipos de $A A C$ en el predio 1 se definen meses referentes Ro a R5 (Fig. 9):

Ro: Enero, mes de referencia para un consumo mínimo, sin actividad académica, con un valor medio de $4535 \mathrm{KWh}$

- R1: Junio y Agosto, meses de referencia para el funcionamiento a pleno de la sede en días templados y frescos (sin el uso de equipos de AAC y con posible uso de estufas eléctricas solamente en boxes y oficinas) con un consumo medio mensual de $5998 \mathrm{KWh}$

R2: Mayo y Setiembre, meses referentes de un uso mínimo de AAC, entre 3 a 5 días al mes; su consumo medio mensual es de $7018 \mathrm{KWh}$

- R3: Febrero, Marzo y Abril, meses de referencia para el funcionamiento pleno de la sede pero con días calurosos, generando un incremento moderado en el consumo de energía eléctrica debido al uso de equipos de AAC; su consumo promedio es de $9562 \mathrm{KWh}$

- R4: Octubre y Noviembre, meses representativos de mayor consumo para una sede en pleno 
D. Mendoza et al.

funcionamiento con días muy calurosos, dando un promedio de $11245 \mathrm{KWh}$

- R5: Diciembre, mes representativo del funcionamiento de la sede pero sin clases, con días calurosos, dando un consumo medio mensual de $9324 \mathrm{KWh}$

En el mes de Julio se tiene dos semanas de receso (JulioR), por lo que se estima que el consumo debe ser similar a la mitad del mes de enero, es decir $2265 \mathrm{KWh}$, mientras que para las dos semanas restantes el consumo es $2548 \mathrm{KWh}$.

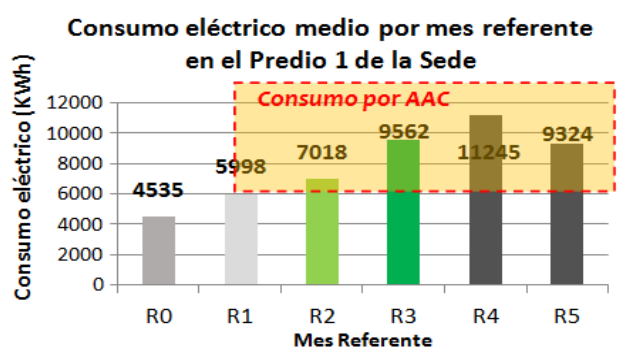

Fig. 9. Meses referentes y consumos medios mensuales estimados por el uso de equipos de AAC en el Predio 1.

En base a dichos meses referentes se identifican las áreas de consumo y los meses de uso de cada área, luego se los relaciona con los consumos medios mensuales del periodo 2013-2018, y finalmente se estima el consumo eléctrico por el uso de equipos de AAC en época estival. Así para el Predio 1 se obtienen los siguientes resultados (Fig. 10):

Consumo eléctrico mensual estimado por el uso de equipos de $A A C$ en pleno funcionamiento (con dictado de clases): $1 \mathrm{MWh}$ a $5 \mathrm{MWh}$

- Consumo eléctrico mensual estimado por el uso de equipos de AAC sin dictado de clases, sólo exámenes finales y actividad administrativa: 3,3 MWh

- Consumo eléctrico anual estimado por el uso de equipos de AAC: $26,5 \mathrm{MWh}$, lo que representa el 28 \% del Consumo Medio Anual del Predio 1.

Consumo mensual estimado por el uso de AAC en el Predio 1

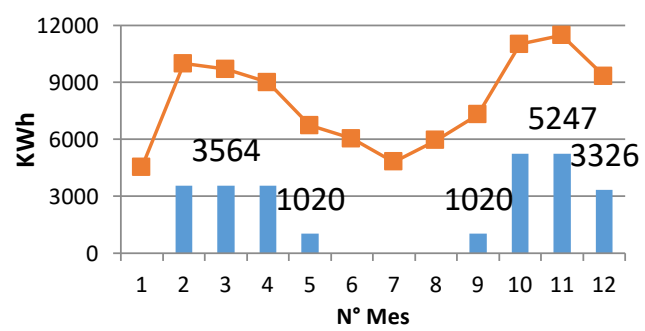

Fig. 10. Estimación anual del consumo eléctrico por el uso de equipos de Aire acondicionado (AAC) en el Predio 1.

4.6 IMPACTOS LOCALES: CONFORMACION DE ESPACIOS CONFORTABLES Y DESCONFORTABLES
Para afrontar las largas jornadas de actividad académica en época estival se hace uso intensivo de los equipos de AAC: todos los ambientes se climatizan durante todo el día. Esta modalidad genera la conformación de (Fig. 11):

- Espacios Cerrados Confortables (ECC): aulas, oficinas, salones, laboratorios y boxes con temperaturas entre $23{ }^{\circ} \mathrm{C}$ y $26^{\circ} \mathrm{C}$

- Espacios Abiertos Desconfortables (EAD): pasillos abiertos y pequeños patios (en donde se encuentran instalados los intercambiadores de los equipos de $A A C$ ) que absorben radiación durante el día y lo ceden al atardecer, dando lugar a temperaturas mayores a $40{ }^{\circ} \mathrm{C}$.

b)

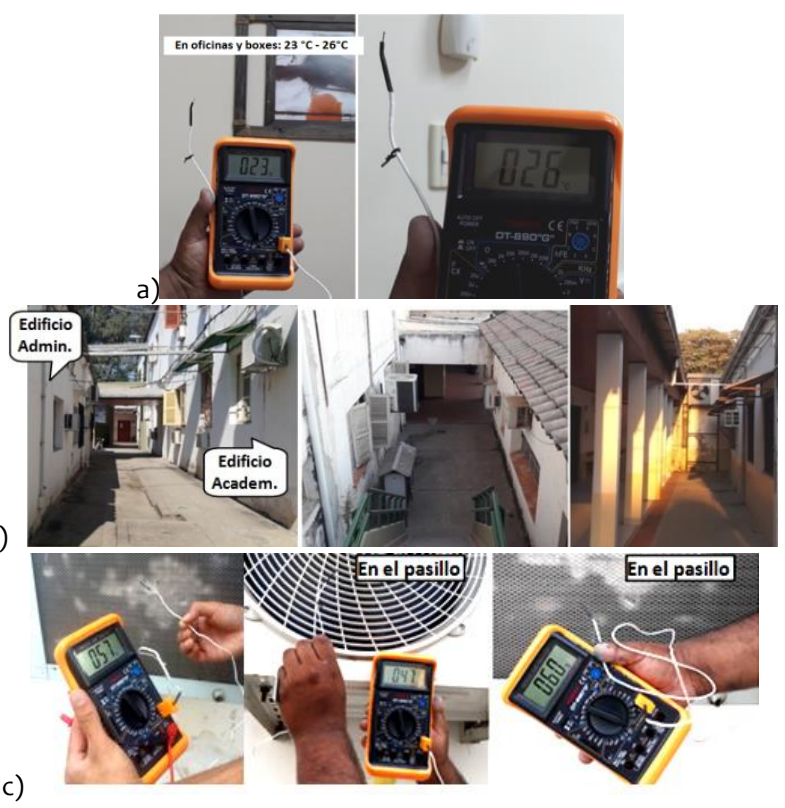

Figura 11 - a) Espacios Cerrados Confortables con $23^{\circ} \mathrm{Ca} 26^{\circ} \mathrm{C}$ en el edificio administrativo; b) Espacios Abiertos Desconfortables (pasillos y patios internos con alta circulación); c) Temperaturas mayores a $40^{\circ} \mathrm{C}$ registradas en los pasillos y a la salida de los intercambiadores de los equipos de equipos de aire acondicionado.

Como los ECC y EAD se comunican directamente, las personas se desplazan entre ambos espacios, pasando de ambientes confortables a ambientes desconfortables, con saltos de temperaturas de más de $15{ }^{\circ} \mathrm{C}$. Esto impacta negativamente en la salud de la comunidad generando malestar e incomodidad.

\subsection{AUSENCIA DE ESPACIOS VERDES EN EAD}

Los espacios verdes y la existencia de plantas en el predio son muy escasos. Los patios tienen pasillos de cemento, algo de césped y tierra. La ausencia de canteros con plantas es total.

\subsection{HUELLA DE CARBONO POR EL USO DE EQUIPOS DE AIRE ACONDICIONADO}

Aplicando el procedimiento de la norma PAS 2050:2011 y utilizando un Factor de emisión de 0,486 
D. Mendoza et al.

[14], se obtiene que, para un Consumo Medio Anual de 26,5 MWh por el uso de AAC, las Emisiones Anuales en el Predio 1 es de aproximadamente 13 tnCO ${ }_{2}$ eq. Una manera de compensar estas emisiones sería disponer de terrenos con árboles adultos: entre 1300 y 2600 unidades para absorber el $\mathrm{CO}_{2} /$ año.

\section{CONCLUSIONES}

La ciudad de Orán se caracteriza por periodos estivales muy largos (mediados de setiembre a abril) con frecuentes jornadas calurosas (altas temperaturas y humedades relativas); según la norma IRAM 11603 se encuentra en zona bioclimática IIb. Los días frescos e invernales son escasos.

- El edificio público es una Sede Universitaria con dos predios: el Predio 1, unidad de estudio, está situado en zona céntrica.

Las soluciones tecnológicas para el logro de confort térmico en época estival son principalmente los equipos de AAC: absolutamente todos los ambientes del Predio 1 disponen de por lo menos un equipo.

Los ambientes confortables resultan indispensables para las actividades académicas.

De acuerdo al funcionamiento de la Institución educativa (febrero a diciembre) es posible identificar áreas de consumo eléctrico y estimar el consumo eléctrico por el uso de los AACs.

- El Consumo Eléctrico Medio Anual del Predio 1 considerando el periodo 2013-2018 es de 96 MWh; con ello es posible estimar el consumo anual para los próximos años en contextos normales y especiales (ej: por pandemia la institución estuvo cerrada, reduciendo el consumo anual al 57 \%).

El Consumo Eléctrico Anual Estimado por el uso de equipos de $A A C$ en el Predio 1 es de 26,5 MWh, lo que representa el $28 \%$ del Consumo Medio Anual del Predio 1.

El uso de equipos de $A A C$ en el Predio 1 genera la conformación de Espacios Cerrados Confortables (con temperaturas de $23{ }^{\circ} \mathrm{C}$ a $26{ }^{\circ} \mathrm{C}$ ) y Espacios Abiertos de Descarga (con temperaturas mayores a $40{ }^{\circ} \mathrm{C}$ durante toda la tarde) que son altamente transitados, generando malestar e incomodidad en la comunidad.

Los espacios verdes en el Predio 1 son escasos.

Las Emisiones Anuales Estimadas de $\mathrm{CO}_{2}$ por consumo eléctrico de $A A C$ en el Predio 1 es de aproximadamente $13 \mathrm{tnCO} \mathrm{CO}_{2} \mathrm{eq}$, valor que podría ser compensado con la disponibilidad de 1300 a 2600 árboles adultos.

Reflexión: La compensación de emisiones no debería ser la solución al problema del calentamiento global y cambio climático. Resulta necesario aplicar estrategias de prevención y reducción. Un rediseño bioclimático del Predio 1 contribuiría a prevenir y reducir las emisiones de $\mathrm{CO}_{2}$ por el consumo eléctrico para el logro del confort térmico (uso de equipos de AAC) y otras demandas eléctricas, y compensar las emisiones que no se pudieron evitar.

\section{REFERENCIAS}

[1] I. Ballarini y V. Corrado, " Application of energy rating methods to the existing building stock: analysis of some residential buildings in Turin”, Energy Build., vol. 41, no. 7, pp. 790-800, jul. 2009.

[2] I. Blasco Lucas, "Aportes de la arquitectura sustentable en el sector residencial sobre el balance energético-ambiental argentino", AVERMA, vol. 12, pp. 07.17-07.24. 2008.

[3] D. Y. Goswami, "A review and future prospects of renewable energy in the global energy system", en Proceedings of ISES World Congress 2007 (Vol. I - Vol. V), Ed. D.Y. Goswami and Y. Zhao, Germany: Springer, pp. 3-10.2008

[4] Fundación Ck-12 (2016, Jan 17). El efecto invernadero [Online] Available: https://www.ck12.org/book/ck-12-conceptos-de-cienciasde-la-tierra-grados-6-8-en-espa\%c3\%b1ol/section/7.13/

[5] IPCC (2007, 2018). Cambio climático 2014 - Informe de síntesis [Online]. Available: https://www.ipcc.ch/site/ assets/uploads/2018/02/SYR_AR5_FINAL_full_es.pdf

[6] WRI (2019, Set). WRI lidēra la Iniciativa Edificios Cero Carbono para Todos lanzada en la Cumbre de Acción Climática de la ONU [Online]. Available: https://www.wri.org/news/2019/og/release-wri-leads-zerocarbon-buildings-all-initiative-launched-un-climate-action.

[7] S. Du Can y L. Price, "Sectoral trends in global energy use and greenhouse gas emissions", Energy Policy, vol. 36, no. 4, pp. 13861403, apr. 2008.

[8] PNUD (2020, Set 28). Programa de las Naciones Unidas para el Desarrollo en Argentina. [Online] Available: http://www.ar.undp.org/. [9] SAyDS, “Tercera Comunicación Nacional de la República Argentina a la Convención Marco de las Naciones Unidas sobre el Cambio Climático, Secretaría de Ambiente y Desarrollo Sustentable de la Nación", 2015

[10] R. Harduim y J. S. Cárcamo Pérez J.S. (2013). Sustentabilidad y Desarrollo. Ed. Universidad Nacional de Moreno [Online]. Available: http://www.unmeditora.unm.edu.ar/files/sustentabilidad-web.pdf.

[11] G. Tapia, "La huella ecológica y las estrategias organizacionales", XXXVII Jornadas Nacionales de Administración Financiera, Set, 2017.

[12] Noticias ONU (2020, set), Noticias ONU [Online]. Avalilabe: https://news.un.org/es/story/2020/09/1480142.

[13] National Geographic (2020, May). La disminución de las emisiones de carbono, por las restricciones de COVID-19, no apaciguará el cambio climático [Online]. Available: https://www.nationalgeographicla.com/ciencia/2020/05/

disminucion-de-las-emisiones-de-carbono-no-apaciguara-el-cambioclimatico.

[14] MAPBA (2017). Cálculo de la huella de carbono [Online]. Available: https://www.gba.gob.ar/static/agroindustria/

docs/direccion_de_sustentabilidad_medio_ambiente_y_cambio_cli

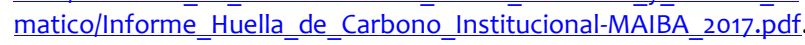
[15] S. B. Jacob, J. L. Strack, J. C. Branda, G. J. Murcia y J. A. Suárez, "Evaluación del consumo eléctrico en el sector residencial de Mar del Plata. Aspectos económicos y ambientales", AVERMA, vol. 17, pp. 01.35-01.42, 2013.

[16] Dirección de Sustentabilidad, Medio Ambiente y Cambio Climático (DSMAyCC), "Manual de Aplicación de la Huella de Carbono", Ministerio de Agroindustria (Prov. de Buenos Aires), 2018. [17] Convención Marco de las Naciones Unidas sobre Cambio Climático (CMNUCC), Naciones Unidas ONU, Nueva York, EEUU, 1992. [18] SMN (2020, Set). Servicio Meteorológico Nacional [Online]. Available: https://www.smn.gob.ar/.

[19] CRC-SAS (2020, Set). Centro Regional del Clima del Sur de América del Sur [Online]. Available: https://www.crcsas.org/es/monitoreo_por_ciudades.php. 
D. Mendoza et al.

[20] N. Sarmiento Barbieri, S. Belmonte, P. Dellicompagni, J. Franco J y K. Escalante, "Atlas de Radiación Solar de la Provincia de Salta. Sistema de Información Geográfico Digital”, Grupo Planificación Energética y Gestión Territorial, Instituto de Investigaciones en Energía No Convencional, CONICET- U.N.Sa, Salta, Argentina, 2017.

[21] E. N. Correa, S. Flores Larsen y G. Lesino, "Isla de calor Urbana: efecto de los pavimentos. Informe de avance", AVERMA, vol. 7, no. 2, 2003.

[22] H. A. Luna. Conociendo lo Nuestro. Orán. Argentina, 2000. 